\title{
Impacto das Redes de Negócios para Startups: Um Estudo Empírico na IETEC/CEFET-RJ
}

\author{
Alternative Title: The Impact of Business Networks for Startups: An Empirical \\ Study at IETEC/CEFET-RJ
}

\author{
Rafael E. L. Escalfoni \\ CEFET-RJ Nova Friburgo \\ Av. Gov. R. Silveira, 1900 \\ Nova Friburgo, RJ, Brasil \\ rafael.escalfoni@cefet-rj.br
}

\author{
Marcelo A. S. Irineu \\ CEFET-RJ Maracanã \\ Av. Maracanã, 151 \\ Rio de Janeiro, RJ, Brasil \\ marcelo.irineu@cefet-rj.br
}

\author{
Jonice Oliveira \\ PPGI - UFRJ \\ CCMN, Cidade Universitária \\ Rio de Janeiro, RJ, Brasil \\ jonice@dcc.ufrj.br
}

\begin{abstract}
RESUMO
As startups têm ganhado papel de destaque no cenário atual devido a seu desempenho para o lançamento de inovações com baixo custo e risco. Contudo, para assegurar o sucesso desses empreendimentos é necessário estabelecer um ambiente colaborativo para incentivar e dar suporte às atividades. Compreender e sistematizar tais requisitos do ecossistema de startups é fundamental para estabelecer maior capacidade competitiva. O presente artigo apresenta um estudo empírico dos aspectos das diferentes estruturas de colaboração na incubadora IETEC CEFET-RJ.
\end{abstract}

\section{Palavras-Chave}

Ecossistemas de Startups; redes de negócios; inovação.

\begin{abstract}
Startups companies have prominent role in the current business scenario. This is because they can get innovation with reduced costs and risks. However, to ensure the success of these ventures it is necessary to establish a collaborative environment to encourage and support those activities. Understanding and systematizing such startup ecosystem requirements is critical to establishing greater competitive capacity. This paper presents an empirical study of different structures of the collaboration aspects in incubator IETEC CEFET-RJ.
\end{abstract}

\section{CCS Concepts}

-Information systems $\rightarrow$ Expert systems; Social networks;

\section{Keywords}

Startup Ecosystem; business networking; innovation

Permission to make digital or hard copies of all or part of this work for personal or classroom use is granted without fee provided that copies are not made or distributed for profit or commercial advantage and that copies bear this notice and the full citation on the first page. To copy otherwise, or republish, to post on servers or to redistribute to lists, requires prior specific permission and/or a fee.

SBSI 2017 June $5^{\text {th }}-8^{\text {th }}$, 2017, Lavras, Minas Gerais, Brazil

Copyright SBC 2017.

\section{INTRODUÇÃO}

Startups são empreendimentos associados à inovação e tecnologia que vêm ganhando papel de destaque no cenário atual em função da flexibilidade do modelo de negócios, baixos investimentos iniciais e maior dinamicidade nos ciclos de transferência tecnológica [10]. Esta estrutura de negócios exerce um papel fundamental no lançamento de novos produtos em um ambiente no qual a complexidade tem aumentado consideravelmente e despertado a necessidade de buscar novas estratégias para geração de inovações e diferenciais competitivos [7].

O estabelecimento de parcerias é essencial para a geração de inovações, pois o sucesso depende de um conjunto de infraestruturas e capacidades que abarcam desde a concepção do produto até a comercialização e distribuição no mercado. Além disso, a colaboração entre organizações é uma importante forma de lidar com as incertezas e riscos inerentes ao processo de inovação $[5,15]$. Frequentemente, empresas consolidadas não controlam esses ativos complementares, e, no caso das startups, tal necessidade fica mais latente [4].

As startups têm maiores chances de sucesso quando se estabelecem em ecossistemas empreendedores, que estimulem o desenvolvimento de negócios e inovações [12]. Os ecossistemas de startups são núcleos tecnológicos, onde parceiros compartilham recursos e habilidades específicas, através de apoio mútuo para estabelecer maior capacidade de competitividade em um cenário maior [6]. Desta forma, entender como tais relações de parcerias entre atores do ecossistema é crítico tanto da empresa quanto do próprio ambiente. O presente artigo apresenta um estudo feito na incubadora tecnológica do CEFET-RJ, a IETEC, onde foram identificados aspectos de colaboração entre os diferentes atores participantes deste ambiente de negócios, destacando os principais recursos compartilhados e auxiliando na identificação das características dos relacionamentos entre empreendedores.

O restante deste artigo está organizado da seguinte forma: a Seção 2 apresenta os conceitos que fundamentam este trabalho. A Seção 3 detalha a caracterização da pesquisa - a elaboração do questionário utilizado, da IETEC e perfil dos entrevistados. A Seção 4 traz uma discussão sobre as respostas obtidas. Na Seção 5 são apresentadas as conclusões da pesquisa e os trabalhos futuros. 


\section{FUNDAMENTAÇÃO TEÓRICA}

\subsection{Startups}

O termo "startup" é utilizado para designar uma instituição temporária formada com o intuito de validar conceitos até que se tenha as condições mínimas para operacionalizar uma empresa, um projeto de organização que visa identificar um modelo de negócio consolidado e que possa ser expandido [3]. Caso não haja sucesso em encontrar este caminho, a natureza do empreendimento permite interrompê-lo sem maiores prejuízos aos colaboradores e demais envolvidos, o que permite enfrentar maiores riscos e incertezas [10].

As novas ferramentas e metodologias de desenvolvimento, como o movimento Lean Startup, propõem uma metodologia de experimentação e aprendizagem interativa. Seu ciclo de vida envolve um conjunto de passos que engloba desde o desenvolvimento de um conceito até o estabelecimento do negócio. Segundo Santos [11], as ações podem ser agrupadas nas seguintes fases:

- Descoberta: agrupa as ações para validar as hipóteses formuladas e para verificar se a solução ataca um problema relevante que consiga atrair o interesse das pessoas;

- Validação: uma vez que o problema fica bem definido na etapa anterior, busca-se conhecer o segmento de clientes dispostos a pagar pelo produto. São feitos os ajustes necessários para tornar o produto rentável. Por vezes, recorre-se a ajustes para atender melhor às necessidades, estratégia conhecida como pivotagem.

- Criação do Cliente: até este ponto, o produto já foi desenvolvido e seu público-alvo já está bem definido. A criação do cliente está relacionada com a massificação do produto e a fidelização, o esforço para popularizar a marca.

- Construção da Empresa: este estágio é a consolidação do empreendimento, quando a startup se torna um negócio. Há uma estruturação da organização, com a definição dos papéis e responsabilidades.

Este tipo de empreendimento, tem sido tratado com grande expectativa por conta de seu potencial para o lançamento de inovações disruptivas [14]. Devido a sua dinâmica, os custos de lançamento de produtos tornaram-se muito menores, trazendo à tona outros fatores de maior importância como por exemplo a inventividade e conhecimento de seus idealizadores e maior tolerância a riscos.

Como forma de viabilizar tais negócios, um número crescente de instituições de apoio tem se estabelecido para orientar o negócio nos seus primeiros dias. São investidores e capitalistas de risco, incubadoras e aceleradoras, além de órgãos governamentais de suporte - atores que compõem os ecossistemas de startups, provendo infraestrutura, espaço de mercado, parcerias consolidadas e processos estabelecidos [1].

\subsection{Ecossistemas de Startups}

Ecossistema de startups são arranjos de atores (empreendedores, apoiadores, universidades, dentre outros) que buscam estabelecer os meios necessários para lançar novos produtos no mercado [13]. Essa comunidade funciona de maneira harmônica e dinâmica, tal qual um ecossistema biológico, onde o meio está constantemente se adaptando às mudanças, como quando ocorre extinção de algum componente [13].

A rede de negócios é um dos aspectos principais para o cenário das startups. O compartilhamento de ideias, experiências entre empreendedores novatos e experientes auxilia no aprendizado e desenvolvimento de competências essenciais para o sucesso de empresas. As conexões com organizações de suporte, como universidades e órgãos de fomento permitem acesso a recursos complementares para o desenvolvimento de produtos, tais como tecnologias e linhas de financiamento [8] [9] [13].

Para assegurar o equilíbrio e, consequentemente seu êxito em apoiar startups, diferentes tipos de parcerias devem ser firmadas. Motoyama e Watkins [9] estabelecem quatro conexões chaves para o ecossistema de startups, são elas:

- Conexões entre empreendedores: estabelecem uma comunidade de aprendizagem, onde as interações possibilitam a troca de saberes entre empreendedores com diferentes níveis de experiência. Os feedbacks obtidos por meio deste tipo de rede proporcionam um meio de aprendizado fundamental sobre o negócio.

- Conexões entre organizações de suporte: compreendem as relações estratégicas e funcionais entre instituições de pesquisa, universidades, incubadoras, dentre outras. Determinam o direcionamento dado ao ecossistema através da promoção de temas de interesse e no suporte de empreendimentos relacionados.

- Conexões entre empreendedores e principais organizações de apoio: Representam as parceiras firmadas entre startups e as organizações de suporte.

- Demais conexões de apoio: Evidenciam outros arranjos existentes entre empreendedores e atores diversos, como mentores, fornecedores e demais empresas parceiras.

\section{ABORDAGEM METODOLÓGICA}

O presente estudo recorreu a evidências baseadas na experiência de especialistas com diferentes perspectivas de um ecossistema de startups. Participaram através de questionários e entrevistas três gestores de empresas intensivas em conhecimento, um representante de um órgão de fomento e o coordenador de incubadora.

No centro da aplicação do estudo encontra-se a IETEC a incubadora de empresas tecnológicas do CEFET-RJ. Fundada em 1994 com a proposta de apoiar empreendimentos na área de telecomunicação, desenvolvimento de hardware e software, a instituição passou por uma reformulação em 2010, passando a contemplar projetos tecnológicos e inovadores de todas as áreas de conhecimento mantidas pelo CEFET-RJ. Atualmente, a IETEC possui 8 projetos residentes, além de ter contribuído para a graduação de 18 empresas com um faturamento médio de $\mathrm{R} \$ 6,5$ milhões.

\subsection{Processo Investigativo}

As entrevistas foram apoiadas por roteiros semiestruturados, constituídos de perguntas principais baseadas nas dimensões propostas por Motoyama e Watkins [9] que evidenciam a relevância de diferentes conexões dentro do ecossistema, sobretudo as interações entre empreendedores e demais participantes do ambiente de negócios, dentro e fora da incubadora. 
Para isto, foram elaboradas questões abertas que buscavam caracterizar o ecossistema e identificar a relevância das relações:

1. Conexões entre empreendedores: (a) Quem são os principais parceiros? (b) O que leva a buscar cooperação? (c) Como são formadas e mantidas as parcerias? Há um processo formal de identificação de colaboradores? (d) Que tipo de recurso é compartilhado? (e) Que critérios são adotados para aceitação de parceiros?

2. Conexões entre empreendedores e principais organizações de apoio: (a) Quais as principais motivações para buscar apoio de instituições de suporte? (b) Quais são as organizações de fomento mais importantes para o seu negócio? (c) Como tais órgãos têm auxiliado o seu negócio?

3. Demais conexões de apoio: (a) Seu negócio mantém vínculos com outras organizações? (b) Qual é o papel destas parcerias?

4. Conexões entre organizações de suporte: (a) Possui parcerias com outros órgãos de apoio (quais)? (b) O que se busca neste tipo de parceria? (c) Como funcionam tais conexões?

O tratamento de dados foi feito utilizando o método de Análise de Conteúdo, no qual buscou-se identificar padrões que possam surgir dos documentos analisados [2]. O processo de análise foi conduzido em 3 etapas:

- Pré-análise: em um primeiro momento, o material das entrevistas e dos questionários foi organizado através de uma categorização. Para isto, as questões foram divididas nos seguintes assuntos principais: caracterização do agente participante, formação e prospecção de parcerias, percepção da importância de conexões formadas com empreendedores e instituições de apoio, procedimentos para a manutenção e estratégias de negócio voltadas para as redes, papel e relevância das instituições apoiadoras para o negócio. As respostas obtidas foram separadas pelo perfil dos especialistas, levando em conta sua experiência empreendedora e o papel exercido na organização estudada.

- Descrição analítica: neste passo, buscou-se elucidar as opiniões a partir da construção de quadro de referências e da busca por sínteses coincidentes e ideias divergentes. Contrapomos as opiniões para identificar conflitos e então voltamos a procurar os participantes para tirar dúvidas; e

- Interpretação: após tirar dúvidas, foram identificadas as conexão entre ideias e conhecimentos adquiridos ao longo do trabalho. A análise crítica destas relações e a base teórica que permeia este trabalho, formam a base das evidências empíricas deste estudo.

\section{RESULTADOS OBTIDOS}

Conforme identificado pela pesquisa, as conexões entre empreendedores manifestam-se de diversas maneiras: através da trocas informais de conhecimento por meio do espaço de co-working, por reuniões periódicas na incubadora e relações pontuais decorrentes de indicações. As interações, normalmente ocorrem para troca de experiências, para obter conhecimentos complementares ou para solucionar gargalos em processos operacionais. A literatura indica como uma das razões deste tipo de cooperação, a complementaridade da cadeia de valor [4]. Conforme identificado, há relatos de parcerias firmadas com empresas consolidadas para este fim.

No ano passado, iniciou-se um programa de mentoria. Os projetos que participaram da dinâmica, passaram por um processo de compreensão dos propósitos de seus produtos e então foi feita a escolha do mentor mais adequado para cada caso. Empreendedores novatos passaram a se reunir com empresários experientes periodicamente por meio de videoconferências. Este contato possibilitou melhorar a percepção do negócio, acarretando em reformulações de conceitos ou mesmo dando mais confiança ao conceito do produto. Alguns projetos montaram parcerias e conseguiram aportes financeiros e apoio por meio de consultorias.

Com relação às conexões entre organizações de apoio, verificou-se um ambiente fértil em ações para prover uma estrutura adequada para os empreendedores. A exemplo das manifestações, a IETEC está envolvida em consórcios entre incubadoras e parques tecnológicos e fóruns temáticos para promoção de inovação, onde tem participado ativamente de programas para intercâmbio de experiências. A instituição tem mantido parcerias com empresas, sobretudo as empresas graduadas, que atuam ativamente no programa de mentoria.

O relacionamento entre empreendedores e organizações de apoio ocorre predominantemente por meio da incubadora. Porém, grande parte dos empreendedores mantém um vínculo sistemático com universidades e centros de pesquisa. O CEFET-RJ, por exemplo, possui um núcleo de inovação tecnológica, responsável por promover a inovação e transferência tecnológica, além de servir de interface da universidade com a incubadora.

Os empreendedores têm conseguido aporte financeiro por meio de fomento. Para facilitar o acesso à informação, a IETEC tem mantido um mapeamento de editais e das políticas propostas dos órgãos de fomento. Este recurso tem sido usado para promover o alinhamento das startups com as aspirações da Sociedade manifestadas pelas linhas de financiamento disponibilizadas.

O processo investigativo também almejou identificar as principais barreiras para a formação de parcerias. Verificouse que a falta de planejamento é um problema recorrente, que dificulta a comunicação com os pares e a percepção de futuro para o negócio. O medo em expor as opiniões pode comprometer as relações: por vezes, o empreendedor tem receio de expor as ideias e ser plagiado - faltam mecanismos para distinguir as informações sensíveis para o negócio das que devem ser compartilhadas com os potenciais parceiros. Os problemas de comunicação dos interlocutores da startup também foram relatados, assim como a falta de informações sobre potenciais parceiros, a falta de sinergia e problemas financeiros.

\section{CONCLUSÃO E TRABALHOS FUTUROS}

A inovação tem sido descrita como o único caminho para estabelecer um modelo de competividade sustentável na economia atual [14]. Neste contexto, as startups têm sido uma poderosa ferramenta de experimentação e aprendizagem, com um grande potencial para a geração de produtos com maior valor agregado. Contudo, o sucesso desse tipo de empreendi- 
mento é dependente do ecossistema no qual está inserido e das relações mantidas entre seus agentes [3][4][9].

O presente artigo apresenta um estudo em andamento que busca compreender como ocorrem as interações entre atores de ecossistemas de startups para aprimorá-las. Nesta primeira etapa da pesquisa, utilizou-se a IETEC/CEFETRJ como objeto de estudo, onde foi feito um levantamento de como as relações ocorrem atualmente, vislumbrando oportunidades de aperfeiçoamento por meio de técnicas e ferramentas computacionais.

Os resultados obtidos através do estudo não podem ser generalizados, mas trazem indícios de que as conexões chaves mencionadas por Motoyama e Watkins [9] estão sendo contempladas de alguma forma. Por outro lado, deve-se considerar o grau de sistematização das interações e a dependência dos agentes principais, sobretudo da incubadora. Não há um processo estruturado para a identificação e gestão da rede e todas as atividades ocorrem de maneira ad hoc, o que pode deixar grandes oportunidades dependentes da percepção dos envolvidos. É preciso criar mecanismos para difundir melhor as informações sobre os recursos disponíveis na rede, a exemplo do que foi feito com o processo de acompanhamento e divulgação dos editais de fomento mencionado.

Em trabalhos futuros, esperamos ampliar nosso conhecimento acerca do universo das startups por meio de uma análise investigativa mais detalhada. Estamos planejando um estudo envolvendo outras incubadoras, aceleradoras e outros parceiros da IETEC. Pretendemos explorar o planejamento de parcerias. O objetivo é auxiliar os participantes do ambiente empreendedor a terem uma visão mais ampla das possibilidades de parceria e promover a gestão de rede por meio de mecanismos de análise de redes sociais. Um exemplo de aplicação seria a identificação dos agentes principais da rede e os gargalos para cada participante. Também vislumbramos a utilização de sistemas de recomendação para identificar parceiros mais apropriados.

\section{AGRADECIMENTOS}

Gostaríamos de agradecer à Incubadora de Empresas Tecnológicas IETEC CEFET-RJ por todo apoio ao projeto. Ao CNPq, CAPES e FAPERJ.

\section{REFERENCES}

[1] S. D. Anthony. The new corporate garage. Harvard Business Review, 1(1):1-11, September 2012.

[2] L. Bardin. Análise de Conteúdo. Edições 70, Lisboa, Portugal, 2009.

[3] S. G. Blank and B. Dorf. The startup owner's manual: the step-by-step guide for building a great company. K\&S Ranch Publishing Division, Pescadero, 2012.

[4] A. Cândido and C. Souza. A adoção de práticas de inovação aberta pelas pequenas e médias empresas: um estudo empírico. Revista Tecnologia e Sociedade, 11(23):1-20, 2015.

[5] H. Chesbrough and M. Appleyard. Open innovation and strategy. California management review, 50(1):57-76, 2007.

[6] D. Cukier, F. Kon, and N. Krueger. Towards a software startup ecosystems maturity model, 2015.

[7] J. Hardwick, A. Anderson, and D. Cruickshank. Trust formation processes in innovative collaborations.
European Journal of Innovation Management, 16(1):4-21, 2013.

[8] D. Isenberg. Babson entrepreneurship ecosystem project.

[9] Y. Motoyama and K. Waltins. Examining the connections within the startup ecosystem: A case study of st. louis, 2014.

[10] E. Ries. The Lean Startup. Crown Business, 1 edition, 2011.

[11] M. C. F. R. Santos. O ecossistema de startups de software da cidade de São Paulo, 2015.

[12] B. Spigel. The relational organization of entrepreneurial ecosystems. Entrepreneurship Theory and Practice, June 2015.

[13] N. N. J. Torres and C. R. B. de Souza. Uma revisão de literatura sobre ecossistemas de startups de tecnologia. In Proceedings of the XII Brazilian Symposium on Information Systems, pages 385-392. Federal University of Santa Catarina - UFSC, May 2016.

[14] T. Weiblen and H. Chesbrough. Engaging with startups to enhance corporate innovation. California Management Review, 57(2), 2016.

[15] J. West and M. Bogers. Leveraging external sources of innovation: A review of research of open innovation. The Journal of Product Innovation Management, 31(4):814-831, 2014. 